\title{
Recent Image Compression Strategies to Reduce the Cost of Transmission in Wireless Sensor Networks
}

\author{
Benfilali Mostefa ${ }^{1}$, Gafour Abdkader ${ }^{2}$, Boukli H. Sofiane ${ }^{2}$ \\ Bechar University ${ }^{1}$ \\ Djillali LIABES University ${ }^{2}$
}

\begin{abstract}
The main obstacle to transmit images in wireless sensor networks is the lack of an appropriate strategy for processing the large volume of data such as images. The high rate packets errors because of what numbers very high packets carrying the data of the captured images and the need for retransmission in case of errors, and more, the energy reserve and band bandwidth is insufficient to accomplish these tasks. This paper presents new effective technique used the technique Background subtraction to compress, process and transmit the images in a wireless sensor network. The practical results show the effectiveness of this approach to make the image compression in the networks of wireless sensors achievable, reliable and efficient in terms of energy and the minimization of amount of image data.
\end{abstract}

\section{Introduction}

Wireless Sensor Networks (WSN) was initially motivated by military applications for battlefield surveillance [1], [2]. In recent years, the usage of WSN in civilian situations, such as disaster detection, health care systems, environment monitoring, weather forecast system, and habitat monitoring [3,4]. A largescale deployment of sensors can be used to collect physical and environmental information. Still images taken by digital cameras, which are one kind of sensors, are very useful for presenting remote on-site views in surveillance applications such as fire prevention systems and damage observation applications. Due to the inherent characteristics of WSN such as loss-prone, low-bandwidth and orderchanging transmission of packets, the question of how to provide reliable, short delay image transmission over WSN becomes a demanding and interesting research topic. Images are extensively used for monitoring in surveillance applications. With the help of remotely located digital cameras, the host application can get on-site views from faraway places. Due to the fact that image files are usually large in size and the number of images being transmitted is numerous, the transmission of images takes up a great portion of traffic over the networks. Traditionally, images are transmitted over reliable, high-bandwidth wired links. Therefore, the duration between an image being requested and finally being displayed is quite short and almost negligible to the user. However, if images are transmitted over a wireless channel, for example, the error-prone, order changing and lossy wireless link, the duration will have to be prolonged and images may not be displayed smoothly to the end user [5].

The image sensor nodes have the following characteristics and requirements [6]:

1. Higher processing power and more memory Image acquisition and processing are compute and memory intensive tasks due to the high information content of image.

2. Real-time and high communication bandwidth Real-time is a requirement for image sensor network. To reduce the requirement of high communication bandwidth, image compression and partial image analysis need to be implemented at the node. Through simple image analysis, only necessary information is transmitted to the base station.

3. Since energy of each image sensor node is supplied with a limited energy battery, low power design technology must be considered. Image transmission consumes more energy than computation. Energy efficient image transmission scheme is needed.

4. Robust transmission and quality of service (QoS): Due to the wireless channel is prone to be interfered by noise, the failure of information transmission often occurs. Robust transmission strategies are needed to resolve the problem.

5. Distributed processing and collaboration: each image sensor node captures the image in its own view. Due to fault consideration or densely deployment, the field of view of neighbor image sensor nodes may overlap. Collaborative processing is needed to reduce the redundant.

The goal of this paper is to provide an image compression technique in wireless sensor networks, for a monitoring system that can minimize to the maximum number of transmission of images captured at nodes sinks, since much of network energy is wasted only for emissions and receptions. We try to use a technique called "background subtraction" , which uses the reference image of the area to be monitored, and the current image (real time), the difference between these two images that can be exploited in the Detection of objects in the area to be monitored. 
This paper is organized as follows: Section II presents some related works. Section III brings the statements and definitions of the proposed approach. Simulation results are presented in Section IV, followed by conclusions and references.

\section{Related Work}

Image compression is a well-established research field, but sensor networks present a context in which new design issues have to be addressed. The main characteristic of such networks is nodes with scarce resources. In fact, the primary focus on energy, computational power and allocated memory call for new approaches. In the literature of WSNs, several compression algorithms have been proposed. The image compression techniques and processing algorithms in a wireless sensor network are classified in two categories [7]:

\section{$\checkmark \quad$ Local processing and compression; \\ $\checkmark$ Distributed processing and compression.}

\subsection{Local processing and compression}

The processing of data at the node source is also necessary to provide for possible loss of information during the transmission of packets to the sink, and to control the quantity of data to be sent (at the same time, the quality of the image) according to the state of the network.

There are several compression algorithms in the literature. Some may provide high compression rates, but they cannot be implemented in sensors nodes networks, because of resource limitations on sensor nodes. In [8] presented a study to evaluate the performance of several traditional image compression algorithms on a sensor node. They studied five algorithms: JPEG2000, Sub Sampling SS, DCT, SPITH and JPEG. The results show that for JPEG2000, DCT, SMITH and JPEG, the energy cost of processing is higher than the transmission cost of the image without compression. The results show that Sub Sampling (SS) is the unique simplest implementation algorithm and presents energy savings with respect to the no compression case, allowing a power reduction of about $29 \%$ for the considered application.

The advantages of local data compression are:

$\checkmark$ Prolongation of the lifetime of the source node.

$\checkmark$ The reduction in the amount of data circulating on the network.

$\checkmark$ Packet loss tolerance.

Recent studies have shown that the transmission of images without compression through the current WSNs is still very costly in energy $[9,10]$. Cristian
Duran-Faundez and al measured in [11] the consumption of a node MICA2 of Crossbow linked to a Cyclops camera when acquiring and transmitting an image of size $128 \times 128$ ( 8 bits per pixel). The node has consumed $91 \mathrm{~mJ}$ excluding headers of packets while the camera had about $90 \mathrm{~mJ}$ energy consumption. This consumption is very high for the WSN, which rapidly depletes the node battery $[11,12]$. According to high energy cost of transferring entire images through the network, compression becomes a necessity.

In the literature, some local processing of proposals to avoid the implementation of a complex load distribution between several nodes of the network. The compression at source has certain advantages. Indeed, it can extend the network life; special attention should be taken to avoid draining the battery quickly the source node. Local compression also allows contributing to the decrease the congestion in network. Among the implemented algorithms based on local processing and compression are as follows: JPEG2000, JPEG, SPIHT, DCT and DWT [19].

Compression by the jpeg technique is a method more used in image compression literature, but this technique is not adapted to be used by the sensor nodes because the nodes of the sensors suffer from some constraints such as, lack Energy, memory space, computing power, the problem of the jpeg technique is related in the step of computing DCT (Discrete Cosine Transform) factors. Standard JPEG consists to divide the image into blocks of $\mathrm{NxN}$ pixels (generally of the blocks of $8 \times 8$ or $(16 \times 16)$ and to apply to each block: the DCT. The blocks are then quantized and coded with RLE and Huffman [13]. All steps of the JPEG algorithm are DCT factors that require more calculations, which lead to greater energy consumption.

Some research groups use techniques based on local compression, some work can be cited:

Kaddachi and al. [14] benefited from the attractive features of the DCT Loeffler (execution speed and reduced computational complexity) to propose a compression technique at source dedicated to WSNs. The disadvantage of this approach is the additional cost of a possible integration of IP (Intellectual Property) developed within the nodes of WSN.

Makkaoui and al. [15] have proven the effectiveness of the zonal DCT for implementation on a wireless sensor network source node. This technique significantly reduces the computational complexity of the conventional DCT without significantly degrading the image quality at the reception. Despite the low complexity of the zonal DCT compared to conventional DCT, the complete compression chain (DCT quantization zonal + entropy coding + packetization) has considerable complexity. The energy savings achieved could be improved by a hardware implementation of this approach. 
JPEG2000 [16] is an image compression algorithm based on a very complex process that includes a dyadic wavelet transform, a bit allocation, a quantization and entropy coding. Wu and Abouzeid [26] proposed a technique based on the JPEG 2000 standard, but which respects the demanding requirements of networks of vision sensors. At first, the idea that the processing cost was negligible prevailed, but this statement is certainly wrong [12]. For the platform mentioned in [8], JPEG2000 did not give good results.

\subsection{Distributed processing and compression}

Due to the distributed nature of the sensor networks, it was evident that many of the work would be based on distributed compression of the images. There are several published proposals based on distributed compression, even if they do not really realize distributed processing, but rather collaborative processing.

In the bibliography of image sensor networks, two different approaches have been classified as "distributed" methods. The first class (most used) takes advantage of the existing correlation between a sequence of images captured by two different cameras (same capture area), seeking to reduce the amount of data to be transmitted from each. A second class considers an approach that uses exactly a distributed processing, sending some areas of an image to different nodes so that they each make a part of the processing.

We will describe and discuss some works found in the literature on the distributed processing of images applied specifically to networks of wireless sensors.

In [17] the authors proposed a scheme for multiple cameras that capture different points of view of the same scene. Here a camera node takes an image of a general scene and a set of secondary cameras each have an overlapping view of the region of the first scene. The method is based on the form context approach presented in [bb]. But this approach has the disadvantage because is the additional cost of energy and processing to detect the overlap between the capture of the nodes, in addition, Congestion in the network due to sending more packets.

In [16] the authors proposed a technique based on distributed image compression using the JPEG2000 standard. The basic idea is to distribute the processing load of the computation of factors the wavelet transforms between the different nodes. Two techniques for the exchange of data have been proposed:

Method of parallel wavelet transform: In the first method, the application of a parallel wavelet transform [18] is proposed. The captured image is divided into $n$ data blocks $\mathrm{R}_{1}, \ldots, \mathrm{R}_{\mathrm{n}}$, consisting of one or more lines. These blocks are transmitted to some neighboring nodes of the source.

$\checkmark$ Tiling Method: In this second method, the captured image is divided into tiles (of data blocks), and then these tiles are distributed to a number of neighboring nodes. These nodes perform the 2-D wavelet transformation independently on these tiles. Finally, the results are transmitted to an aggregate node.

In [20] the authors presented a distributed compression scheme based on LBT (Lapped Biorthogonal Transform), an algorithm of zero-tree type (which exploits the parent-child relationship between the different coefficients) and Golomb coding. The LBT requires much less computation and memory than the DWT.

All its approaches to distributed processing (distributed data compression) have not been validated experimentally on real platforms of wireless sensor networks. The theoretical results presented in his references are good, but the studied models should be confronted with satisfactory experimental results [11].

This paper presents a technique for image compression in a wireless sensors network; we consider the nodes are static position. All previous studies are based on the set of nodes to perform image compression tasks. We try to present a technique based on the method of "background subtraction" with image quantization, this approach saves considerably the node energy equipped with a camera in the network, thus reducing the number of transmission of Images in the network since the operations of the transmission and the reception are two extremely energy-intensive operations and consequently minimizing the complexity of the processes that enter into the image compression operation.

\section{Proposed system}

Our approach is based on an image compression technique using an object detection method, called background subtraction, we justify our choice of this technique because in the case of image sequences, it is possible to increase the compression rate, consider the fact that between two consecutive images most pixels practically retain the same value. Because, generally an image belonging to images sequence, the bottom of the decor is very often fixed and only some elements have changed from an image to the next. In addition, a big part of the various elements between two consecutive images of a sequence can be assigned to a local displacement or not the objects. Compression techniques seek to transmit only the difference between two successive images [21]. The goal is to minimize the maximum size of the compressed image, minimize the number of sending packets of compressed images in the network and 
saves lot of energy of node equipped of camera as well as reduces the network communication overhead, which leads to an increase of the network lifetime to accomplish the task image compression.

Background Subtraction technique [21] is an image processing technique used to determine Changes between two or more images. The difference between the reference image $\mathbf{I}_{\mathbf{R}}$ and current image $\mathbf{I}_{\mathbf{C}}$ is calculated by finding the difference between each pixel in each image, and generating an image as a result.

Background subtraction is particularly a commonly used technique for motion segmentation in static scenes [21]. The objective of this technique is to detect moving regions by the subtraction between the current image "IC" pixel by pixel and the reference image " $\mathbf{I}_{\mathbf{R}}$ ". The reference image " $\mathbf{I}_{\mathbf{R}}$ " is captured and sending to the processing center, while another copy is saved at each node during the network initialization phase. If the difference between the pixels is higher than a threshold $\mathbf{T}$, then $\mathbf{T}$ is classified as a foreground. The image reference $\mathbf{I}_{\mathbf{R}}$ is updated with new images over time to adapt to dynamic scene changes [21].

There are different approaches in literature using the background subtraction technique to detect the foreground regions and to update background. In [23] uses the simple version of this scheme where a pixel at location $(\mathrm{x}, \mathrm{y})$ in the current image ( $\left.\mathbf{I}_{\mathbf{C}}\right)$ at time $\boldsymbol{t}$ is marked as foreground if:

$$
\mid \mathrm{I}_{\mathrm{C}}(\mathrm{x}, \mathrm{y})-\left(\mathrm{I}_{\mathrm{R}}(\mathrm{x}, \mathrm{y}) \mid>\mathrm{T}\right.
$$

The equation is satisfied where "Ț" is a predefined threshold. The background image "I $\mathbf{I}_{\mathbf{R}}$ " is updated by the use of an Infinite Impulse Response (IIR) filter as follows:

$$
\mathrm{I}_{\mathrm{Rt}}+1=\alpha \mathrm{I}_{\mathrm{Ct}}+(1-\alpha) \mathrm{I}_{\mathrm{Rt}}
$$

The reference background is updated with new images over time to adapt to dynamic scene changes. In literature, several methods to update the reference image or background image among these techniques are found in [23, 24].

The purpose of our system sent only changes in the reference $\mathbf{I}_{\mathbf{R}}$ image, the objects detected instead of sending the entire image. The changed pixels compared to the reference image are extracted from the current image at time " $t$ " that are higher than the threshold value $\mathbf{T}$.

These small image (the difference) is quantified with coordinates), compressed by method discussed in [27] and sending by the sensors nodes with their coordinate values to the destination (the processing centre). In destination (the processing centre), the final image is reconstructed using an Image Reference $\mathbf{I}_{\mathbf{R}}$ sent during the initial phase to the destination.
This technique is very simple and easy to implement while satisfying the image quality requirement and decrease the transmission rate of the captured images. Simulation results show that our system increases the lifetime of the network up to 10 times with respect to the transmission of the image without using without using our approach.

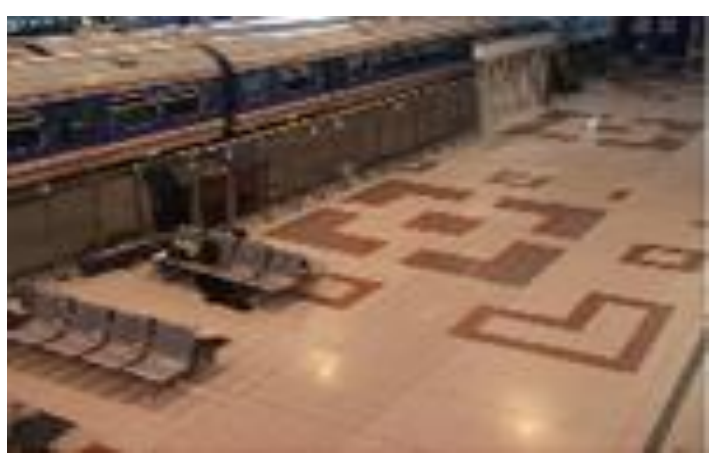

Figure 1. Reference Image $\mathbf{I}_{\mathbf{R}}$.

The network consists of a set of nodes, each node equipped with a camera contains a memory used to store reference $\mathbf{I}_{\mathbf{R}}$ image in the initial deployment phase; the image shown in Figure 1, also transmitted to the destination (the processing centre), for reconstructing the final image. After, de-quantified and de-compression operations.

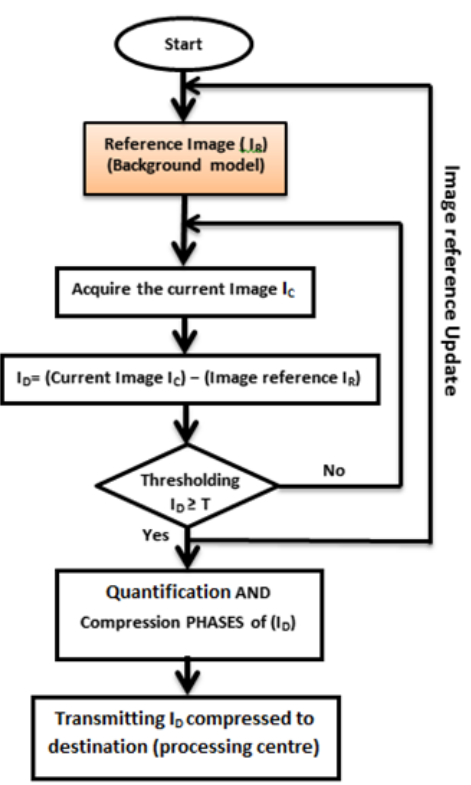

Figure 2. Organization chart of local processing at node

The system detects of objects in $\mathrm{I}_{\mathrm{C}}$ by subtracting the current image (I) from the reference image $\left(\mathbf{I}_{\mathbf{R}}\right)$, after, if the value of the subtraction operation is greater than a threshold $\mathbf{T}$, Firstly, going to the quantification / compression phases by (distributed compression operation discussed in [27]) of this difference, Secondly, updating the reference image, 
by remplacing the reference image by current image $\mathbf{I}_{\mathbf{C}}$ in the local node.

But, in the processing center the update of the reference image $\mathbf{I}_{\mathbf{R}}$ is replaced by the reconstructed image, else the system go to a new capture current image Ic, finally, sent this compressed difference to distination (processing center), the steps is detailed in following organization chart 1 .

The structure of the system shown in Figure 3 is divided into three zones:

1) The capture area: where nodes occupied a camera to capture images, after the compression phase, using the proposed method;

2) Nodes medium area: Consists of intermediates nodes, its roles are to transmit the compressed image to the destination;

3) Destination area: where decompression is applied according to the method proposed in [3].

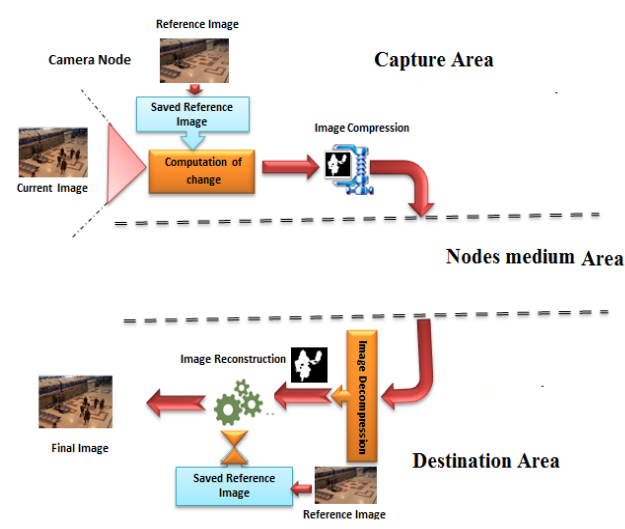

Figure 3. System Structure

After the camera node deployed in the network, if there is no change in the new captured image or in the current image $\mathbf{I}_{\mathbf{C}}$ compared to the reference image $\mathbf{I}_{\mathbf{R}}$, the system switches to recapture, else, if there is a change as shown in Figure 4.

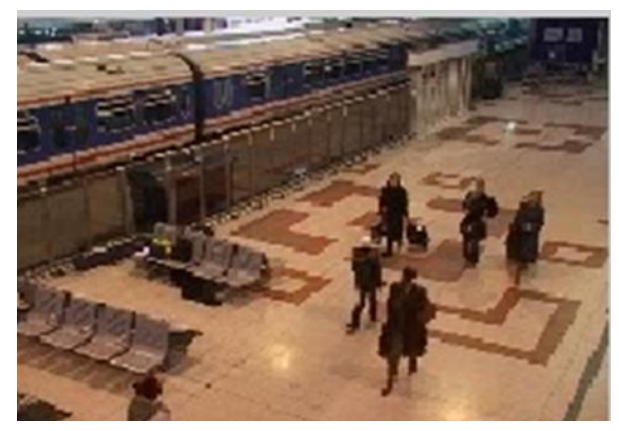

Figure 4. Current Image (IC)

Thus, instead of sending the entire captured image, only changed pixels are returned after compressed by the method discussed in [27]. The image of Figure 5 is the image obtained after the subtraction operation between the current image $\left(\mathrm{I}_{\mathrm{C}}\right)$ shown in Figure 4 and the reference image shown in Figure 1.

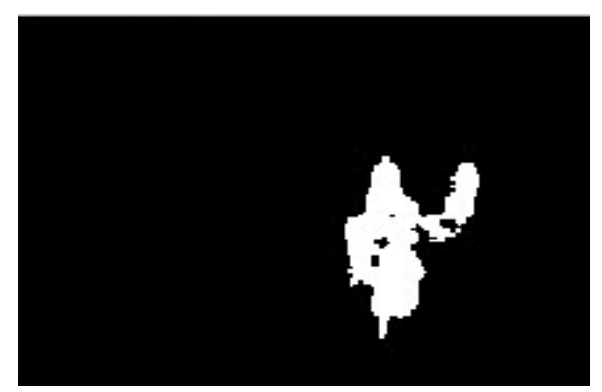

Figure 5. Image ID obtained from $\left(I_{C}-I_{R}\right)$

Finally, image difference $I_{D}$ obtained at the node equipped with a camera is illustrated in Figure 6, after, cropping the size $75 / 75$ area of the image. Image compression solves the problem of the size of the image, which reduce the amount of data required to represent an image.

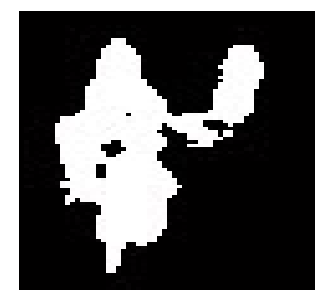

Figure 6. Image difference ID after cropping

If the difference $I_{D}$ contains black pixels or a few pixel values less than a threshold " $T$ " fixed in advance, the system has nothing, he must move to another capture, otherwise, the difference between $\mathrm{I}_{\mathrm{C}}$ and $I_{R}$, the captured image and the reference image their sizes are 75 / 75 area, must now be passed to compression phase discussed in [27] and the quantization phase of the small image illustrated in figure 7 , also leads to reduce the size of this difference image.

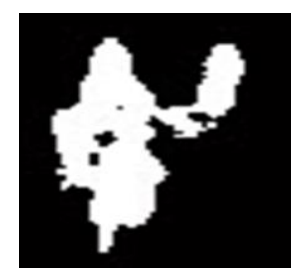

Figure 7. Quantized Image

The image Compressed get in the final phase must be remitted to the destination or (the processing centre). The compression rate is increased by more than 75 times is depended the detected changes. Finally, in destination (Processing Centre), the final 
image is reconstructed using the De-compressed image received and De-quantization, finally, the image is saved to the destination.

\section{Simulation and results}

The performance of wireless sensor network is measured by the life of the network, which is measured from when the network starts communication operation until the first network node fails due to lack of energy). The energy consumed for receiving a bit is:

$$
\mathrm{E}_{\mathrm{RX}}=\varepsilon_{\mathrm{e}}
$$

The energy consumed to transmit a bit is:

$$
\mathrm{E}_{\mathrm{TX}}=\varepsilon_{e}+\varepsilon_{x} \mathrm{k}^{\mathrm{x}}
$$

$\varepsilon_{\mathrm{e}}$ is the energy consumed by the circuit for each bit, $\varepsilon_{\mathrm{x}}$ is the energy dissipated for each bit by meter, $\mathrm{k}$ is the distance between a transmitter and a receiver wireless, and $2 \leq \alpha \leq 4$ is the parameter loss path [28]. The energy consumed to accomplish our proposed technique is:

$$
E_{\text {cost }}=\beta
$$

$\beta$ is the energy consumption for the following operations: Determine the difference between $I_{R}$ and $\mathrm{I}_{\mathrm{C}}$ and quantification.

The communication range of node $\mathrm{k}$ is chosen from 5 to 20 meters while $\alpha$ is chosen 2 .

The captured image or the current image is of size $547 \mathrm{~kb}$, jpeg format and a resolution is $1600 \times 900$ as shown in Figure 4, which reduces to $30 \mathrm{~kb}$, after calculating the change by comparing the reference image of Figure 1 and the current image that is shown in Figure 4. Since there is only a small change represented by the figure 5 cropped of image is reduced to $6 \mathrm{~kb}$ and after the application of quantification reduced to 800 bytes therefore the compression ratio is more than 50 times, although, wavelet compression requires much more processing power and high battery.

The parameters for the wireless communication energy model are typically equal respectively $\boldsymbol{\varepsilon}_{x}=100$ $\times 10^{-12}$ and $\varepsilon_{e}=50 \times 110^{-9}$ as shown in [16]. The range communication $\mathrm{k}$ of node is selected between 4 and 30 meters, and $\alpha$ chosen equal to 3 .

Simulation is realized by MATLAB 12, the figure 8 and figure 9 respectively shows the relationships of energy consumption image compressed using our approach in figure 9 and in Figure 8 without the use of our approach while the distance from 4 to 30 meters. Energy consumed without the use of our method is .03uj while the energy consumed by using our method is 0.00003 uj. Thus, more than 80 times, the life time of the network is increased.

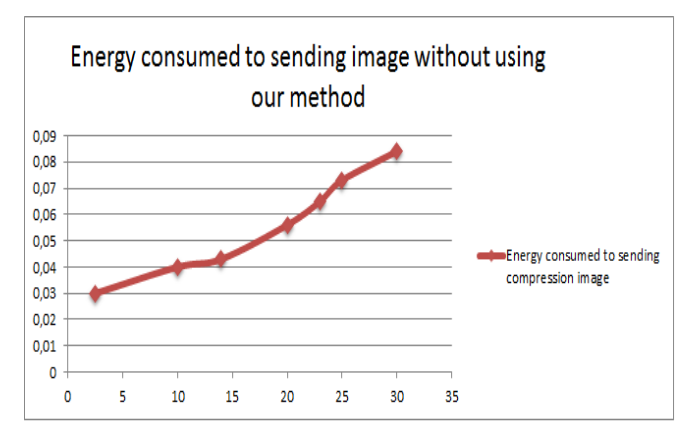

Figure 8. Energy consumption $(\mu \mathrm{j})$ in relation to distance source in meter.

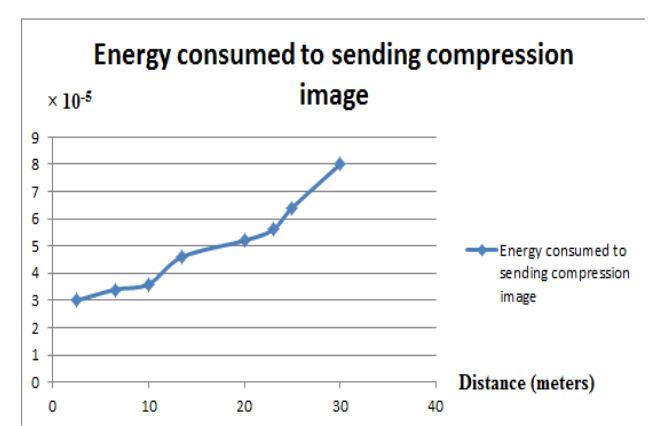

Figure 9. Energy consumption $(\mu \mathrm{j})$ in relation to distance source in meter

\section{Conclusion}

In this paper, we present a novel technique of image compression used the background subtraction applied to a wireless Image Sensor Network. Our approach has proven their performance compared to other methods such as compression image distributed, since our approach requires very low processing power and a very low consumption rate of the energy. We determined the different changes each time between two images, reference image and the current image, and keeps only the difference, at the same time our method minimize sends images in the network, since the operation of the transmission and reception very expensive in energy, for this we have increased the maximum duration of node and network life.

Therefore, the compression ratio is very high because the difference extract is very small compared to the current image that contains the background and foreground, that is, some changes in the reference image.

We showed by simulation that our approach improves the energy efficiency of the node camera sensor networks. The proposed technique is very simple and easy to implement and gives better results, since not demand processing power as compression methods. Performance evaluation has shown that our approach increased the node lifetime equipped camera with a more than $50 \%$. 


\section{References}

[1] H. Salemand, N. Mohamed, "Middleware challenges and approaches for wireless sensor networks", IEEE Distributed Systems Online, 7(3):1, 2006.

[2] R. Kay, F. Mattern, “The design space of wireless sensor networks", IEEE Wireless Communications, 11(6):54-61, 2004

[3] A.Bharathidasan and V.A.S.Ponduru. Sensor networks:Anoverview. IEEE Potentials, 22(2): 20-23, 2003.

[4] N. Xu, "A Survey of Sensor Network Applications", http://courses.cs.tamu.edu/rabi/cpsc617/resources/sensor\% 20nw-survey.pdf.

[5] YanDu, "A Progressively Reliable Image Transport Protocol over Wireless Sensor Networks", Thesis submitted to the Faculty of Graduate and Post-doctoral Studies In partial fulfillment of the requirements For the M.Sc. degree in Computer Science, (CYan Du,Ottawa, Canada, 2008.

[6] Zhi-Yan Cao, Zheng-Zhou Ji, Ming-Zeng Hu,"An Image Sensor Node for Wireless Sensor Networks", IEEE, Information Technology Coding and Computing (ITCC'05), April 2009.

[7] Min Wu, Chang Wen Chen, "Multiple bitstream image transmission over wireless sensor networks", In: Sensors, October 2003, Proceedings of IEEE (Vol. 2, pp. 727-731). IEEE.

[8] Ferrigno, L., S. Marano, V. Paciello, A. Pietro santo (2005). "Balancing computational and transmission power consumption in wireless image sensor networks", In: IEEE International Conference on Virtual Environments, HumanComputer Interfaces, and Measures Systems (VECIMS 2005), Giardini Naxos, Italy.

[9] Huaming Wu, Alhussein A. Abouzeid, "Error resilient image transport in wireless sensor networks", Elsevier, Computer Networks, 2006; 50:2873-2887.

[10] Min Wu, Chang Wen Chen, "Multiple bitstream image transmission over wireless sensor networks", In: Sensors, October 2003, Proceedings of IEEE (Vol. 2, pp. 727-731). IEEE.

[11] Cristian Duran-Faundez, "Transmission of images on wireless sensor networks under the constraint of energy", $\mathrm{Ph}$.D. dissertation, Henri Poincary University, Center of Research in Automation of Nancy, June 2009.

[12] Vincent Lecuire, Cristian Duran-Faundez, Nicolas Krommenacker, "Energy-efficient transmission of waveletbased images in wireless sensor networks",EURASIP J. Image Video Process 2007; Article ID. 47345, 11 pages.

[13] K. S. Thyagarajan, "Still Image and Video Compression with MATLAB", Published by JOHN WILEY \& SONS, INC, 2011, ISBN 978-0-470-88692-2, 442 pages.
[14] Kaddachi Lassaad, Soudani Adel, Lecuire Vincent, Tourki Kholdoun, Mekkaoui Leila, Moureaux Jean-Marie, "Low power hardware-based image compression solution for wireless camera sensor networks", Computer Standards and Interfaces, 2012; 34:14-23.

[15] Mekkaoui Leila, Vincent Lecuire, Jean-Marie Moureaux, "Camera Sensor Networks", Published in the 2nd International Conference on Image Processing Theory, Tools and applications, IPTA 2010.

[16] C.A. Christopoulos, T. Ebrahimi, N. Skodras, "JPEG2000: the new still picture compression standard", In Proceedings of the 2000 ACM workshops on multimedia. ACM Press. Los Angeles, California, United States. pp. 4549.

[17] R. Wagner, Robert Nowak, R. Baraniuk, "Distributed image compression for sensor networks using correspondence analysis and super-resolution", In: Proceedings of 2003 International Conference on Image Processing (ICIP). Vol. 1. pp. 597-600.

[18] Marino, Francesco Maria, Vincenzo Piuri and Jr. Earl E. Swartz lander (1999). A parallel implementation of the 2-D discrete wavelet transform without interprocessor communications. IEEE Transactions on Signal Processing, 47(11), 3179-3184.

[19] CHEFI Ahmed, "Design of a micro CMOS image sensor with low energy consumption for networks of wireless sensors", doctoral thesis, the EEATS Doctoral School, University of GRENOBLE, 28/01/2014.

[20] Qin Lu, Wusheng Luo, Jidong Wang, Bo Chen, "Low complexity and energy efficient image compression scheme for wireless sensor networks", Elsevier, Computer Networks, 2008; 52 (13): 2594-2603

[21] Jean-Paul Dubus, "Compression of static and dynamic images, Technical journal of engineer", Date of publication: 10 March 1996.

[22] McFarlane N, Schofield C., "Segmentation and tracking of piglets in images, Machine Vision Appl 1995; 8(3): 187-193.

[23] McFarlane N, Schofield C. Segmentation and tracking of piglets in images, Machine Vision Appl 1995; 8(3): 187193.

[24] Binnie T. d., Thomson M. S and Wan C. L, Development O A Real Time Image Analysis System For Trafic Monitoring Application", Knowledge Based Systems For Civil And Structural Engineering, pp. 189-195, 1993.

[25] Ali A T., Bulas-Cruz J., Dagless E. L., "Real-Time Motion Detection and Tracking" Proceedings of the 8th Scaninavian Coference On Image Analysis: Tromsoe, mai 25-28, vol. 9612, n. 1, pp. 515-522, 1993.

[26] H. Wu, A.A. Abouzeid, "Energy efficient distributed JPEG2000 image compression in multihop wireless networks" ASWN 2004. pp 152-160. 
[27] W. B. Huang, W. Y. Su, Y. H. Kuo, "VLSI implementation of a modified efficient SPIHT encoder", IEEE Trans. Fundamentals., vol. E89-A, no.12, pp. 3613622, Dec. 2006.

[28] H. Salem, N. Mohamed, "Middleware challenges and approaches for wireless sensor networks", IEEE Distributed Systems Online, 7(3):1, 2006. 